\title{
Los epigramas de Juan Ginés de Sepúlveda. En torno a la atribución de los elogios de Antonio de Nebrija y de Juan Domínguez de Paniza*
}

\author{
RAMÓN GUTIÉRREZ
}

\begin{abstract}
During his lifetime, Juan Ginés de Sepúlveda (1490-1573) only published two epigrams: In Stunicam and In Maximilianum archiducem Austriae. On the basis of an eighteenth-century manuscript, preserved at the Real Academia de la Historia (Madrid), Losada ascribed to Sepúlveda two further epigrams: In Aelium Nebrissensem and In Paniza. The former has certainly to be rejected, taking into account chronological and ecdotic arguments. With respect to the latter (which has to be dated between 1514 and 1522), its ascription to Sepúlveda has to be regarded as a hopeful assumption at best: indeed, even if we lack conclusive arguments for denying Sepúlveda's authorship, conclusive pieces of evidence are missing for supporting such an attribution. A critical edition and translation of the epigrams In Aelium Nebrissensem and In Paniza is also provided.
\end{abstract}

\section{Los traslados de Lamadrid y el Cod. 132 del Real Colegio de España}

En su fundamental monografía sobre Juan Ginés de Sepúlveda, Ángel Losada publicó dos epigramas, atribuyéndolos al humanista de Pozoblanco: uno inédito, In Ioannem Paniza, y otro hasta entonces tenido por obra de un anónimo, In Aelium Nebrissensem. ${ }^{1}$ Losada no editó

* Queremos dejar constancia de nuestro agradecimiento al Prof. Dr. D. Juan J. Gutiérrez Alonso, Rector del Real Colegio de España en Bolonia, por todas las facilidades que ha dado para la redacción del presente artículo. También hemos de agradecer a la Dott.ssa Enrica Coser, encargada del Archivo de la citada institución, por la ayuda prestada. La presente contribución se enmarca en el Proyecto de Investigación "Gramáticos latinos fragmentarios de época imperial (II)" (FFI2017-83315-C2-2-P), dirigido por el Prof. Dr. D. Javier Uría Varela (Universidad de Zaragoza); también él se ha hecho merecedor de mi más sincero agradecimiento, por el interés mostrado en la discusión de las sucesivas versiones de este artículo. Queremos dejar asimismo constancia de nuestra deuda con la Redacción y los evaluadores anónimos de esta revista: sus acertadas sugerencias han contribuido a mejorar sustancialmente este artículo.

1 A. Losada García, Juan Ginés de Sepúlveda a través de su Epistolario y nuevos documentos (Madrid, 1949), 585; véanse asimismo las referencias que Losada hace a estos epigramas en $149-150$ y 384. 
propiamente los mencionados poemas, sino que se limitó a reproducir un documento manuscrito custodiado en la Biblioteca de la Real Academia de la Historia (ms. 9/6117-59; en adelante M). Este contiene el traslado de los mencionados epigramas que en 1792 envió a la Real Academia de la Historia desde Bolonia el colegial Juan José de Lamadrid y Obejo, con el objeto de contribuir a la reedición de las obras de Juan Ginés de Sepúlveda. $^{2}$ De dicho traslado existe copia en el Archivo del Real Colegio de España en Bolonia (serie Miscellanea secc. XVI-XIX, caja 7 "Relazioni, dissertazioni, secc. XVIII [1792] - XIX”, legajo 3, f. 7v-8r; en adelante B). Tanto en el traslado $\mathbf{M}$ como en su copia $\mathbf{B}$, al texto de los epigramas en cuestión le sigue una nota de Lamadrid, con significativas variantes en uno y otro testimonio:

M: Estos dos Epigrammas se hallan escritos de mano de Sepúlveda: el primero, al principio de una Grammática de Ant. ${ }^{\circ}$ Nebrixa, $q^{\mathrm{e}}$ es el libro, que Juan Paniza regaló a la Bibliotheca, y el segundo, al fin del libro del mismo Nebrija - Vafre dicta philosophorum -, el qual está anónimo entre los elogios de Paulo Jovio, Elog. Doct. vir. LXIIII.

B: Estos dos Epigrammas se hallan escritos de mano de Sepúlveda; el primero al principio de la Gramática de Antonio Nebrixa, que es el libro que el S. or Paniza regaló a la Bibliotheca del Colegio; y el segundo también al principio del Libro de Nebrixa - Vafre dicta Philosophorum - y está anónimo entre los elogios de Paulo Jovio.

La Gramática que menciona Lamadrid constituye la primera unidad codicológica (f. 1r-66v) del Cod. 132 del Real Colegio de España (en adelante, C), ${ }^{3}$ un volumen facticio que reúne, sustancialmente, obras

2 Los datos biográficos relativos a Lamadrid pueden hallarse en A. Pérez Martín, Proles Aegidiana, vol. 3 (Bolonia, 1979), 1746-1749, no. 1316. Las obras completas de Juan Ginés de Sepúlveda habían sido editadas por la Real Academia de la Historia doce años antes: Opera Ioannis Genesii Sepulvedae cum edita tum inedita accurante Regia Historiae Academia, 4 vol. (Matriti, ex typographia Regia de la Gazeta, 1780). Sobre los pormenores de esta edición, véase J. Gil Fernández, "Una labor de equipo: la editio Matritensis de Juan Ginés de Sepúlveda”, Cuadernos de filología clásica 8 (1975), 93-129.

3 Se trata de un ejemplar de Antonio de Nebrija, Gramática castellana (Salamanca, [Juan de Porras], 1492 [USTC 766256]). Véanse A. Odriozola, "La caracola del bibliófilo nebrisense", Revista de bibliografía nacional 7 (1946), 3-114, en concreto 39-40, no. 152; M.A. Esparza Torres, H.-J. Niederehe, Bibliografía nebrisense. Las obras completas del humanista Antonio de Nebrija desde 1481 hasta nuestros días (Amsterdam - Philadelphia, PA, 1999), 48-49, no. 18. 
impresas y manuscritas de Antonio de Nebrija. ${ }^{4}$ Lamadrid, como acabamos de ver, afirma (tanto en $\mathbf{M}$ como en $\mathbf{B}$ ) que el primero de los epigramas que copia (el dedicado a Juan de Paniza) se encontraba "al principio" del mencionado ejemplar de la Gramática castellana; sin duda se hallaba transcrito en el folio $a$ de dicho impreso, que no se conserva actualmente, sin que pueda determinarse en qué momento tuvo lugar su pérdida. ${ }^{5}$ En este sentido, por más que Losada escriba que del epigrama In Paniza "se conserva el original autógrafo del propio cronista al principio de una gramática de Antonio de Nebrija que Juan Paniza regaló a la biblioteca del Colegio de Bolonia," ${ }^{6}$ de tales palabras, a nuestro entender, no puede deducirse que, en el momento en que escribía esas líneas, aún se conservase el folio a del mencionado ejemplar de la Gramática castellana. En efecto: si así hubiese sido, Losada habría editado ambos epigramas a partir de $\mathbf{C}$, y no basándose en $\mathbf{M}$.

A su vez, el impreso Vafre dicta philosophorum del que habla Lamadrid se corresponde con la segunda unidad codicológica de $\mathbf{C}$ (constituyendo sus f. 67r-118v). ${ }^{7}$ El epigrama In Aelium Nebrissensem (el segundo de los transcritos por Lamadrid en $\mathbf{M}$ y B) puede aún leerse en el folio $[a] \mathrm{r}$ del mencionado impreso (que se corresponde con el folio $67 \mathrm{r}$ de la numeración continua C), ${ }^{8}$ hallándose, por lo tanto "al principio" de

4 Para una descripción de este códice remitiremos a A. García García, C. Piana, "Los manuscritos filosófico-teológicos, históricos y científicos del Real Colegio de España de Bolonia", Salmanticensis 14 (1967), 81-169, en concreto 140-143; D. Maffei et al., I codici del Collegio di Spagna di Bologna (Milano, 1992), 459-460; R. Gutiérrez González, "La Repetitio quinta de analogia de Antonio de Nebrija", en M.J. Rodrigo Mora (ed.), Nebrija en Bolonia. V Centenario de las Reglas de orthographia en la lengua castellana (1517) (Bologna, 2019), 61-110, en concreto 61-77. La formación de C, por lo demás, fue relativamente tardía. La primera referencia a este volumen tiene lugar en 1624 y su creación es, en todo caso, posterior a 1534 (probable terminus post quem para la llegada al Colegio de los manuscritos nebrisenses reunidos en $\mathbf{C}$ ); véanse P. Martín Baños, "Los manuscritos de Antonio de Nebrija. Un inventario razonado", Boletín de la Real Academia de Extremadura de las Letras y las Artes 23 (2015), 251-346, en concreto 274, n. 36; Gutiérrez González 2019 (cit. n. 4), 68-72.

5 El impreso de la Gramática castellana comienza en el folio a ii que se corresponde con el folio 1 de la numeración continua de $\mathbf{C}$.

${ }^{6}$ Losada 1949 (cit. n. 1), 384.

7 En concreto, se trata de un ejemplar de Antonio de Nebrija, Vafre dicta philosophorum ([Salamanca: Juan de Porras, c. 1502] [USTC 767883]); véanse Odriozola 1946 (cit. n. 3), 54, no. 175 y no. 176; Esparza Torres, Niederehe 1999 (cit. n. 3), 55, no. 44 y 53, no. 34. Con P. Martín Baños, https://www.corpusnebrissense.com/caracola/vafre/vafre 1502.html, ha de considerarse que estamos ante dos partes de un mismo impreso.

8 Véase la Figura 1. 
dicha obra, como se afirma en B, y no "al fin", como erróneamente se asevera en $\mathbf{M}$.

Esta notable divergencia entre las notas que se encuentran en ambos traslados parece indicar que $\mathbf{B}$ no es una mera copia de $\mathbf{M}$, sino que representa una segunda redacción, en la que Lamadrid corrigió errores presentes en el documento enviado a la Real Academia de la Historia. ${ }^{9}$ Esta circunstancia habrá de tenerse en cuenta a la hora de valorar los testimonios de $\mathbf{M}$ y $\mathbf{B}$ para la constitución del texto del epigrama In Paniza (que, como quedó dicho, solo conocemos gracias a las copias realizadas por Lamadrid).

La conservación en $\mathbf{C}$ del epigrama In Aelium Nebrissensem nos permite, por otra parte, evaluar la exactitud de los traslados de Lamadrid. Para ello transcribiremos el texto presente en $\mathbf{C}$, registrando en aparato las diferencias que presentan $\mathbf{M}$ y $\mathbf{B} .{ }^{10}$

Io. Sepulu. in Aelium Nebris. gramat.

ut Quōdam tacuit latię facundia linguę cum fuit Antonii cęsa manus gladiis

sic scelus hoc solers Antonii cura piauit

Quamqū; prior uitam sustutulit ille dedit.

Tit. Io. Sepulu. in Aelium Nebris. gramat.] C : Ioannes Gennesius Sepulveda in Aelium Nebrissensem M : Joannis Sepulveda in Aelium Antonium Nebrisensem. Epygramma B 1 Quōdam] C : quondam MB 3 piauit] $\mathbf{C}$ : piavit MB 4 Quamqū;] $\mathbf{C}$ : quamque $\mathbf{M B}$ uitam] C : vitam $\mathbf{M B}$ | sustutulit] $\mathbf{C}$ : sustulit $\mathbf{M B}$

Como puede observarse, Lamadrid no ha llevado a cabo en $\mathbf{M}$ y $\mathbf{B}$ una transcripción diplomática del folio $67 \mathrm{r}$ de $\mathbf{C}$, sino que se ha tomado ciertas libertades a la hora de copiar el epigrama. Con todo, es de notar que, mientras se ha creído autorizado a variar a su gusto la praescriptio en $\mathbf{M}$ y $\mathbf{B}$, en estos el texto del poema es sustancialmente el mismo que se lee en C. Dejando, en efecto, de lado ciertas minucias (como regularizar el uso de las mayúsculas, desarrollar abreviaturas o sustituir la $u$ consonántica por $v$ ), Lamadrid solo interviene en el verso 4 para corregir sustutulit en sustulit (aunque quizás lo haya hecho inadvertidamente). Así

9 Así, por ejemplo, B corrige la lectura ofrecida en $\mathbf{M}$ del verso 2 del epigrama In Paniza (el comienzo de dicho verso en $\mathbf{M}$, "muneribus ut nullum", es amétrico; B, sin embargo, transmite "muneribus nullum ut," que sí encaja en el pentámetro).

10 Para la edición y traducción del tetrástico, véase $\$ 3$. 
pues, a la hora de establecer el texto del elogio de Paniza a partir de los traslados de Lamadrid, podremos tener cierta confianza en la fiabilidad del texto poético propiamente dicho, mientras que habrá que poner en cuarentena la literalidad del título que precede a dicha composición.

Está, por otra parte, la cuestión de la autografía de ambos epigramas en $\mathbf{C}$, que parece haber sido, junto con la declaración de autoría en sus respectivas praescriptiones, el principal sustento por parte de Losada para adjudicar la paternidad de aquellos a Sepúlveda. En efecto: en la nota antes transcrita, Lamadrid afirma que "estos dos Epigrammas se hallan escritos [en C] de mano de Sepúlveda." Sobre esta cuestión solo podemos pronunciarnos con certeza en lo que toca al epigrama en honor de Nebrija - el único conservado en el códice boloñés - y la conclusión es negativa: la mano que copia en $\mathbf{C}$ el epigrama In Aelium Nebrissensem es totalmente diferente a la tercera mano de los códices Torrepalma y Regio de la Biblioteca de la Real Academia de la Historia, ${ }^{11}$ que los estudiosos emplean como piedra de toque para identificar los autógrafos de Juan Ginés de Sepúlveda. ${ }^{12}$ Habida cuenta de ello, habrá que poner necesariamente en cuarentena la autografía en $\mathbf{C}$ del epigrama In Paniza, dado que tal afirmación se basa únicamente en el testimonio de Lamadrid (cuya escasa fiabilidad a este respecto acabamos de documentar).

Por otra parte, dado que este erudito - como hemos visto anteriormente - se tomó ciertas libertades a la hora de transcribir las praescriptiones de los epigramas - elementos que constituyen, como quedó ya señalado, el segundo de los sustentos que han venido aduciéndose para atribuir a Sepúlveda estas composiciones -, solo cabría recurrir a criterios internos (de estilo) y externos para tratar de dilucidar su autoría.

\section{La producción poética de Sepúlveda}

Dejando de lado las composiciones poéticas publicadas por Losada, solo se conocen otros dos epigramas de Juan Ginés de Sepúlveda, ${ }^{13}$ y ello

11 Se trata, respectivamente, de ms. 9-29-2/5820 (olim E. 3 Est. 27 gr. 1) y ms. 9-292/5819 (olim E. 2 Est. 27 gr. 1); sobre estos, véase, en particular, J.A. Bellido Díaz, "Indefessae labor limae Sepulvedanae en los libros 19 y 20 de la De rebus gestis Caroli quinti historia", Exemplaria classica 12 (2008), 195-248 (con numerosas fotografías).

12 Para un estado de la cuestión, véase J. Solana Pujalte, Dos traducciones castellanas atribuidas a Juan Ginés de Sepúlveda (Córdoba, 1999), 17-19.

13 Cf. Losada 1949 (cit. n. 1), 149-150; J.F. Alcina, Repertorio de la poesía latina del Renacimiento en España (Salamanca, 1995), 190, no. 415. 
gracias a su inclusión en sendas cartas de su epistolario. ${ }^{14}$ Nos referimos, en concreto, al epitafio de Diego López de Zúñiga y al epigrama sobre la entrada del archiduque Maximiliano de Austria en Valladolid.

La primera de las mencionadas composiciones se halla incluida en Epistolae 2.1 [XVI] = Epistolario 13, carta que ha de fecharse en enero o febrero de $1532 .{ }^{15} \mathrm{Su}$ destinatario es Íñigo López de Mendoza, obispo de Burgos, a quien Sepúlveda escribe desde Roma con motivo del fallecimiento del teólogo y escriturista Diego López de Zúñiga. ${ }^{16}$ Tras llevar a cabo una laudatio del finado, el pozoalbense concluye su misiva con un epigrama, que resume y adorna poéticamente los temas desarrollados en el cuerpo de la carta.

Los ecos clásicos de esta composición son perceptibles y remiten, en primera instancia, a los epitafios de Nevio y de Plauto transmitidos por Aulo Gelio ${ }^{17}$ - textos que sirvieron desde temprano de inspiración a otros poetas humanísticos. ${ }^{18}$ Por lo demás, Sepúlveda reelabora dichos modelos atendiendo a un tópico antiguo, que se erige en hilo conductor de esta breve composición: la deseable conjunción de doctrina y afabilidad, que los antiguos alegorizaban recurriendo a la unión de las Musas y de las Gracias (cf. Quint. Inst. 1.10.21 "denique in proverbium usque Graecorum celebratum est, indoctos a Musis atque a Gratiis abesse”). ${ }^{19}$

14 Las citas de las cartas de Sepúlveda se harán siguiendo la edición teubneriana de J.J. Valverde Abril (ed.), Juan Ginés de Sepúlveda, Epistolarum libri septem (München Leipzig, 2003); en adelante, Epistolae. También haremos referencia a I.J. García Pinilla, J. Solana Pujalte (ed., trad.), Juan Ginés de Sepúlveda, Obras completas. IX. Epistolario, 2 vol. (Pozoblanco, 2007); en adelante, Epistolario.

15 Es la fecha que señala J.J. Valverde Abril, "Hacia una edición del Opus epistolarum de Juan Ginés de Sepúlveda: Inventario", Boletín de la Real Academia de la Historia 200 (2003), 481-541, en concreto 518, no. 83.

16 Los principales datos biográficos de Diego López de Zúñiga pueden verse en W.B. Jones, T.B. Deutscher, "Diego López Zúñiga", en P.G. Bietenholz, T.B. Deutscher (ed.), Contemporaries of Erasmus, vol. 2 (Toronto - Buffalo - London, 1986), 348-349.

17 Gell. NA 1.24.2 y 1.24.3; J. Blänsdorf (ed.), Fragmenta poetarum Latinorum (Berlin - New York, 2011), 69 y 70 respectivamente.

18 Por ejemplo, el epitafio compuesto por Carlo Marsuppini para la tumba de Leonardo Bruni: véase V. Schmidt, “A Humanist's Life Summarized: Leonardo Bruni's Epitaph”, Humanistica Lovaniensia 47 (1998), 1-14.

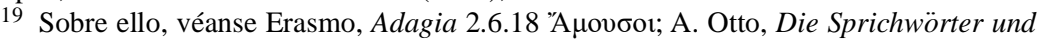
sprichwörtlichen Redensarten der Römer (Leipzig, 1890), 235, no. 1176. 
Transcribimos el epigrama siguiendo la edición teubneriana de Valverde ( si bien regularizamos la ortografía):20

Epigramma

Flete virum, Charites, iacet hic virtutis alumnus

Stunica, saecli laus deliciaeque sui.

Vos quoque lugete, heu, Musae, nam utrumque colebat

ille chorum sancte, gratus utrique fuit,

virtutumque gregi carus: languere videntur

doctrina et probitas, cumque pudore sales.

Tit. epigramma] Sf : epigramma in obitum Iacobi Stunicae $\boldsymbol{\delta} \mathbf{2}$ secli laus] $\mathbf{S f}$ : laus secli $\boldsymbol{\delta}$ $\mathbf{6}$ sales] $\mathbf{S f M}$ : iocus $\mathbf{C}$

Para comodidad de los lectores, ofrecemos la siguiente traducción: ${ }^{21}$

Epigrama. Llorad a un varón, Gracias; yace aquí un discípulo de la virtud: Zúñiga, gloria y deleite de su siglo. Vosotras doleos también, ¡ay, Musas!, pues aquel veneraba santamente uno y otro coro; a uno y otro les fue grato él, querido por la grey de las virtudes: parecen languidecer la doctrina y la honradez, y los donaires junto con el recato.

Se trata, como podemos comprobar, de una composición de cuidada factura y de prosodia irreprochable. En cuanto a la métrica, la única objeción que puede hacerse es que, en el primero de los pentámetros, aparece un monosílabo (laus) al final del primer miembro. De esta anomalía parece haber sido consciente el responsable de la edición

20 Epistolae (cit. n. 14). Las siglas que aparecen en el aparato del epigrama son las siguientes: $\mathbf{S}=$ Io. Genesii Sepulvedae [...] Epistolarum libri septem (Salmanticae, apud Ioannem Mariam da Terranova et Iacobum Archarium, 1557 [USTC 341750]); C = Ioannis Genesii Sepulvedae [...] Opera quae reperiri potuerunt omnia (Coloniae Agrippinae, in officina Birckmannica sumptibus Arnoldi Mylii, 1602 [USTC 2078552]); M = Opera Ioannis Genesii Sepulvedae cum edita tum inedita accurante Regia Historiae Academia (Matriti, ex typographia Regia de la Gazeta, 1780); f = Sevilla, Biblioteca Capitular y Colombina, ms. 59-2-7; $\boldsymbol{\delta}=$ consenso de $\mathbf{C}$ y $\mathbf{M}$.

21 Este epigrama se encuentra traducido en Epistolario 13 (en concreto, p. 47), así como en J.J. Valverde Abril, Io. Genesii Sepulvedae Epistolarum libri VII (Tesis doctoral, Granada, 2001), 62. 
coloniense (C) - con gran probabilidad André Schott, ${ }^{22}$ a quien siguen los editores de $\mathbf{M},-$ quien cambió el orden de palabras del verso 2 del epigrama (saecli laus en laus saecli) para evitar la referida transgresión métrica. Cabe preguntarse si esta enmienda ha de ser aceptada, ${ }^{23} \mathrm{o}$ bien ha de mantenerse el texto presente en la edición salmantina $(\mathbf{S}) .{ }^{24} \mathrm{Si}$ bien esta constituye la fuente última de las ediciones coloniense (C) y matritense $(\mathbf{M}),{ }^{25} \mathrm{y}$, además, nace de un proyecto personal de Sepúlveda, cabe decir que el pozoalbense no participó directamente en el proceso de impresión, del que se encargó su amigo Diego Neila: ${ }^{26}$ y este, en las palabras que dirige al lector antes de la fe de erratas, afirma haber tenido que bregar con un original de imprenta mendoso y con unos tipógrafos

22 Para una primera aproximación a la biografía de este jesuita, puede acudirse a G. Tournoy, “André Schott (1552-1629)”, en C. Nativel (ed.), Centuriae Latinae: Cent une figures humanistes de la Renaissance aux Lumières offertes à Jacques Chomarat (Genève, 1997), 749-753. Como responsable de la edición coloniense de las obras completas de Juan Ginés de Sepúlveda (C) suele señalarse al docto impresor Arnold Mylius, quien fue ciertamente el que sufragó y llevó a cabo la impresión. Ahora bien, de la carta nuncupatoria de esta edición queda clara la intervención en ella de André Schott. En efecto: tras una mención genérica de la Vita Aegidii y del epistolario, Mylius (f. *2v) incide en las traducciones de la Política y de los Parva naturalia de Aristóteles, así como en la traducción del comentario de Alejandro de Afrodisias a la Metafísica - obras que se excluyen de la edición coloniense; a continuación, afirma: "Reliqua vero Sepulvedae monumenta, quae

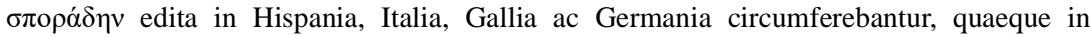
ultimis Hispaniae finibus reperiebantur, a singulari amico, tuique imprimis studioso, And. Schotto, veluti naufragii tabulas collecta ad nosque in Ubios missa et uno fasce colligata, nominis tui auspicio damus" (el dedicatario era Marcus Welser). De esta declaración cabe deducir que fue Schott quien envió a Mylius todos los textos reunidos en la edición coloniense. También ha de atribuirse a Schott la noticia De vita scriptisque Ioan. Genesii Sepulvedae Cordubensis e scriptis eius et epistolis collecta, que figura anónima en los f. $* 3 \mathrm{v}-* * \mathrm{v}$ de la mencionada edición; prueba de ello es que el jesuita belga la insertó en su Hispaniae bibliotheca seu de academiis ac bibliothecis (Francofurti, apud Claudium Marnium et haeredes Ioan. Aubrii, 1608 [USTC 2001679]), 464- 468.

23 Agradezco al Comité Editorial de esta revista el haber suscitado la presente cuestión.

24 La lección secli laus está presente también en f, pero este manuscrito no constituye un testimonio independiente, pues es descriptus respecto de $\mathbf{S}$; véase J.J. Valverde Abril, "La transmisión textual de la epístola latina de Sepúlveda a Felipe II (Sepulv. Epist. 3.6)", en F. Fuentes Moreno, M. del Castillo Herrera et al. (ed.), Quantus qualisque. Homenaje al Profesor Jesús Luque Moreno (Granada, 2016), 625-637, en concreto 631 у 634.

25 Para esta cuestión - que no hemos podido sino presentar en un modo necesariamente reductivo - véase el prefacio de Valverde Abril a Epistolae (VII-XIX); además, I.J. García Pinilla, J. Solana Pujalte (ed.), Juan Ginés de Sepúlveda, Obras completas. VIII. Epistolario (Pozoblanco, 2007), cccxxv-cccxxvii.

26 Sobre Diego de Neila véase B. Cuart, J. Costas, "Diego de Neila, colegial de Bolonia, canónigo de Salamanca y amigo de Juan Ginés de Sepúlveda”, en E. Verdera y Tuells (ed.), El cardenal Albornoz y el Colegio de España, vol. 6 (Bolonia, 1979), 263-313. 
poco diligentes. ${ }^{27}$ Ello hace posible que, ya bien el copista, ya bien los tipógrafos, transpusiesen "laus saecli" en "saecli laus". Para sustentar esta afirmación podemos acudir al estudio métrico que Serrano Cueto hace de los Adagia de Fernando de Arce, impresos en 1533. ${ }^{28}$ Según el análisis estadístico llevado a cabo por el citado estudioso, los monosílabos en final del primer miembro del pentámetro son escasísimos en Fernando de Arce $(1,71 \%)$; de ellos, unos se justifican - siguiendo el uso clásico - por estar precedidos por otro monosílabo $(80 \%)$; mientras que el resto $(20 \%)$ están unidos por aféresis a la palabra que los precede. ${ }^{29}$ Dado que en el presente pasaje no se da ni el uno ni el otro caso, y teniendo en cuenta las deficiencias de la edición salmantina $(\mathbf{S})$ antes referidas, parece sensato adoptar la enmienda laus saecli presente en la edición de Colonia (C).

También en lo que toca al texto de este epigrama, llama asimismo la atención en el verso 5 la lección iocus presente en $\mathbf{C}$ (en lugar del sales transmitido por SfM). La razón de esta variante es difícil de señalar con precisión. Parece, en todo caso, que el autor de esta enmienda pretendió continuar los singulares que antecedían (doctrina, probitas, pudor), que entendía como personificaciones, y que para ello se inspiró en una de las fuentes de esta composición - el epitafio de Nevio (Gell. NA 1.24.3) -, en donde se lee (v. 2-3) "dein Risus Ludus Iocusque / et Numeri innumeri simul omnes conlacrimarunt."

El segundo de los epigramas publicados por Sepúlveda se encuentra en la carta que, desde Pozoblanco, dirigió a Alfonso Guajardo el 31 de octubre de 1548 (Epistolae 4.9[LX] = Epistolario 71). Los hechos históricos que constituyen el trasfondo de esta composición (y que el propio Sepúlveda narra al comienzo del libro XXVI del De rebus gestis Caroli $V$ ) son la entrada de Maximiliano de Austria en Valladolid, el 13 de septiembre de 1548, para contraer matrimonio con su prima María de Austria, hija de Carlos I; el nombramiento de ambos como regentes, el 29 del mismo mes; y la partida del príncipe Felipe (el futuro Felipe II), el 2 de octubre 1548, para iniciar el viaje que le llevaría a Italia, Austria y el Sur de Alemania. Transcribimos el texto del epigrama siguiendo de nuevo

27 S, f. Nnr-v (= Epistolario, Anexo IV): “Quoniam partim amanuensis, qui exemplar scripserat, partim etiam typographorum incuria quibusdam in locis erratum fuisse deprehendimus." Sobre las desventuras de Sepúlveda en lo relativo a llevar a cabo impresiones cuidadas de sus textos, véase J. Gil Fernández, "Introducción histórica”, en García Pinilla, Solana Pujalte 2007 (cit. n. 25), xix-clxix, en concreto lxxxviii-xci.

28 A. Serrano Cueto (ed., trad.), Fernando de Arce, Adagios y Fábulas (Alcañiz Madrid, 2002), lxxxiv-lxxxix.

29 Véase Serrano Cueto 2002 (cit. n. 28), 1xxxviii. 
la edición teubneriana de Valverde Abril (que no registra variantes textuales en la presente composición):

In Maximilianum archiducem Austriae, quo Valdolitum ingresso, mox imber optatissimus, flante Aquilone, decidit. Epigramma Genesii Sepulvedae.

Terra dehiscebat sitiens, fontesque perennes aruerant, maeror cuncta premebat iners.

Te veniente redit facies laetissima rerum imber et optatus flante Aquilone cadit

arridensque tibi vires natura remittit et pluvias infert quo prohibere solet.

Ergo tuus faustum Boreas, dulcissime princeps, adventum signat, cunctaque laeta fore.

Unde venit Boreas, hac te regione profectum, excipimus laeti; tempora laeta dabis,

longaque felici perages cum coniuge felix tempora, sed socero cum seniore senex,

sospite Philippo; quo discedente, feremus maerorem graviter, te remanente, minus.

Ofrecemos - nuevamente en atención a los lectores - la siguiente traducción: ${ }^{30}$

A Maximiliano, archiduque de Austria, tras cuya entrada en Valladolid al punto cayó la ansiadísima lluvia, mientras soplaba el Aquilón. Epigrama de Ginés de Sepúlveda. La tierra se abría sedienta y las fuentes perennes se habían secado; una aflicción estéril oprimía todo. Con tu venida retorna el más alegre aspecto de las cosas y cae la ansiada lluvia mientras sopla el Aquilón; y, sonriéndote, la naturaleza mitiga en consideración hacia ti sus fuerzas y trae las lluvias por medio de aquel con el que suele impedirlas. Así pues, tu Bóreas, dulcísimo príncipe, señala la fausta llegada y que todo ha de ser favorable. De donde viene el Bóreas, llegado de esta región te recibimos alegres; nos darás tiempos alegres y feliz agotarás una larga vida junto con tu feliz cónyuge, ya anciano, pero con un más anciano suegro, estando Felipe

30 Señalamos asimismo las traducciones en Epistolario 71 (en concreto, p. 183) y Valverde Abril 2001 (cit. n. 21), 199. 
sano y salvo: al partir este soportaremos con menor pesar la tristeza, ya que te quedas tú.

Estamos de nuevo ante una composición de claros ecos clásicos, inmediatamente perceptibles en los abundantes préstamos ovidianos. ${ }^{31}$ Más allá de ello, señalaremos brevemente que la vigorosa imagen que abre el epigrama (v. 1 "terra dehiscebat sitiens") tiene una innegable raigambre virgiliana; en nuestra opinión, el pozoalbense se inspira en dos pasajes de las Geórgicas en donde el verbo dehiscere aparece referido a terra: 3.432 "postquam exusta palus terraeque ardore dehiscunt" y 1.479-480 "sistunt amnes terraeque dehiscunt / et maestum inlacrimat templis ebur aeraque sudant." 32 También tiene origen en la literatura clásica el tópico del gobernante que extiende su esfera de influencia a los elementos (v. 3-6), cuyo ejemplo más conocido - y que quizás Sepúlveda tuvo en mente - es el famoso dístico atribuido a Virgilio (Anth. Lat. 256 Riese $=250$ Shackleton Bailey): "Nocte pluit tota, redeunt spectacula mane: / divisum imperium cum Iove, Caesar, habes. ${ }^{33}$ Finalmente, señalaremos que la afirmación (presente en el verso 6) de que el Aquilón impide las lluvias parece inspirada en Sen. Nat. 4b.4.3 "praeterea Aquilone flante aut suum caelum habente minutae pluviae sunt;" a ello, en efecto, apunta que el ablativo absoluto presente en tal pasaje ("Aquilonte flante") aparezca en el verso 4 de este epigrama, si bien con un orden de palabras inverso ("flante Aquilone"), por exigencia del metro.

A la prosodia de esta composición puede hacérsele una única salvedad: en el verso 13 Sepúlveda escande Phïlippō, cuando, en realidad, la

31 En efecto: tres de las siete cláusulas hexamétricas presentes en este epigrama están tomadas literalmente de Ovidio: v. 3 "laetissima rerum" = Ov. Ars 1.359; v. 5 "natura remittit" = Ov. Met. 10.330; v. 11 "coniuge felix" = Ov. Met. 6.681, 7.60, 9.333; Trist. 5.5.21. Sepúlveda llega incluso a tomar de Ovidio el segundo miembro de un pentámetro: v. 12 "cum seniore senex" = Ov. Trist. 2.166 (de donde, a su vez, Mart. Epigr. 6.3.4).

32 En Georg. 3.432, Virgilio describe los efectos del estío; a su vez, en Georg. 1.479480 narra los prodigios que siguieron a la muerte de César. Habida cuenta de la diferencia de contexto, podría pensarse que Sepúlveda no tomó en consideración el segundo de los pasajes citados. Invita, sin embargo, a pensar lo contrario el hecho de que en este epigrama aparezcan elementos que se dan también Georg. 1.479-480; nos referimos, en concreto, a la mención de los cursos de agua (amnes; fontes en Sepúlveda) y al adjetivo maestum, que responde al sustantivo maeror presente en este epigrama.

$33 \mathrm{Si}$ así fuera, Sepúlveda - atendiendo a las circunstancias que pretende reflejar en su poema - habría dado la vuelta al epigrama pseudovirgiliano: la lluvia, entendida ahora como un elemento positivo, es propiciada por la entrada del archiduque Maximiliano en Valladolid. 
primera sílaba es etimológicamente breve ( $P h \check{\imath}$-); se trata, con todo, de un procedimiento común - e incluso banal - entre los poetas humanísticos. ${ }^{34}$

Los dos únicos epigramas publicados por Sepúlveda - separados por tres lustros de distancia - suman veinte versos, que son a todas luces insuficientes para llegar a generalizaciones seguras sobre el estilo poético y la técnica versificatoria del pozoalbense. A este respecto, lo primero que cabría preguntarse es si Sepúlveda da cuenta en algún lugar de su actividad poética. Para responder a ello podemos acudir a la ya mencionada carta a Alfonso Guajardo. En ella, como quedó ya dicho, Sepúlveda envía a su corresponsal el epigrama en honor de Maximiliano de Austria, haciéndolo preceder de las siguientes palabras:

Sepúlveda, Epistolae 4.9(LX).6 = Epistolario 71.3 "Carmen quod in eius [sc. Maximiliani] adventu lusi ad te cum his litteris mitto, ne te lateat me cum Musis mansuetioribus in gratiam redivisse."

$\mathrm{Si}$, como parece dar a entender el contexto, las "musas más apacibles", a las que Sepúlveda retorna, son las de la poesía, ${ }^{35}$ habría de suponerse que, además de los dos epigramas publicados en el Epistolario, Sepúlveda habría compuesto con anterioridad otros que, o bien no salieron de sus cartapacios, o bien tuvieron una circulación muy restringida: y entre ellos podrían, en principio, contarse los epigramas en honor de Nebrija y de Paniza que constituyen el objeto de este artículo.

Por lo demás, cabe preguntarse por qué Sepúlveda solo dio al público dos de sus epigramas. A este respecto hemos de partir de la consideración de que, en el ambiente hispano, ya desde Nebrija el ejercicio de la poesía latina era un inexcusable elemento de prestigio para todo profesional de

34 Sobre los problemas relacionados con la prosodia de los nombres propios en la poesía neolatina, véase especialmente J.M. Maestre Maestre, "En torno a los nombres propios de la poesía latina de los humanistas”, en J. Luque Moreno, P.R. Díaz y Díaz (ed.), Estudios de métrica latina, vol. 2 (Granada, 1999), 549-584.

35 En Cic. Fam. 1.9.23 la expresión "mansuetiores Musae" hace referencia a la filosofía (por oposición a las agrestiores Musae de la oratoria: cf. Cic. Or. 12). Este mismo sentido se testimonia en Sepúlveda, Epistolae 6.6.(LXXXVII).15-16 = Epistolario 114.6 "Atque hanc quidem philosophandi [...] facultatem nobis nostrum Marianum [...] suppeditat [...] Itaque Marianenses hi dies et cum mansuetioribus Musis consuetudo mihi tantopere placent, ut libenter sim omnibus omnes opes concessurus, dum mihi salvo officio liceat hoc modo vivere." En todo caso, frente a este uso ciceroniano, el contexto de la carta a Alfonso Guajardo no permite sino interpretar que las mansuetiores Musae a las que allí se hace referencia son las de la poesía. 
dicha lengua. ${ }^{36}$ Podemos, por otra parte, conjeturar que Sepúlveda fuera consciente de sus limitaciones en este ámbito. En efecto: los dos poemas publicados en su Epistolario son una muestra de su capacidad para componer versos aceptables desde un punto de vista métrico y prosódico, e incluso de su erudición, pero no destacan precisamente por la profundidad de su estro poético. Así pues, el pozoalbense debió juzgar más prudente moverse dentro de una estudiada ambigüedad: $\mathrm{y}$, en tal sentido, mientras en la mencionada carta a Guajardo declara haberse reconciliado con la poesía, nunca publicará más epigramas que los dos contenidos en su Epistolario.

\section{El epigrama en honor de Nebrija}

Según los datos reunidos por Martín Baños, ${ }^{37}$ el epigrama In Aelium Nebrissensem se documenta por primera vez en la edición barcelonesa de 1505 de las Introductiones Latinae de Nebrija, salida de las prensas de Nicolás Spindeler (B). ${ }^{38}$ En el vuelto de su último folio, precedido por un epigrama de ochos dísticos elegíacos con el título Cuiusdam scolaris carmen in laudem Antoni Nebrissensis, ${ }^{39}$ aparece, cerrando el volumen, el epigrama que estamos analizando, con el título Eidem [sic, por "eiusdem"] scolaris carmen tetrasthicon [sic] de eodem; ambas composiciones, como puede verse, se atribuyen a un estudiante anónimo. Martín Baños conjetura que estos poemas se tomaron de la perdida edición barcelonesa de 1500 de las Introductiones Latinae (también de Spindeler), ${ }^{40}$ y que esta última, a su vez, los habría sacado de una edición escolar castellana también perdida. ${ }^{41}$ El tetrástico en honor de Nebrija

36 Sobre la poesía neolatina como actividad de prestigio en Nebrija, véase T. Jiménez Calvente, "Nebrija, poeta áulico: La Peregrinatio Regis et Reginae ad Sanctum Iacobum. Edición, traducción y estudio", Medievalismo 20 (2010), 63-95, en particular 78.

37 P. Martín Baños, Repertorio bibliográfico de las Introductiones Latinae de Antonio de Nebrija (1481-1599) (Vigo, 2014), 330.

38 Antonio de Nebrija, Ars grammatica cum uberrimis commentariis ([Barcelona]: per Nicolaum Spindeler, 1505 [USTC 334592]); véanse Odriozola 1946 (cit. n. 3), 15, no. 18; Esparza Torres, Niederehe 1999 (cit. n. 3), 59-60, no. 61; Martín Baños 2014 (cit. n. 37), 109-110, no. 28.

39 Sobre este epigrama, véase Martín Baños 2014 (cit. n. 37), 327-328.

40 Sobre esta edición, véase Martín Baños 2014 (cit. n. 37), 102, no. 18.

41 Véase Martín Baños 2014 (cit. n. 37), 327 y 330. Por afectar a nuestro razonamiento cronológico, advertimos al lector de la errata presente en la mencionada p. 327, en donde se lee "más bien puede conjeturarse que los contuviera alguna edición escolar castellana anterior a 1505"; Martín Baños ha querido, obviamente, escribir “1500”. 
aparece inmediatamente después en la edición Veneciana de 1512 de las Introductiones de Nebrija (V), que tuvo por editor a Cristóbal Escobar, ${ }^{42}$ y algunos años más tarde "se hizo imprescindible en las ediciones abreviadas granadinas y antequeranas (y por extensión en muchas otras)" de las Introductiones Latinae, figurando siempre en ellas como obra de un anónimo. ${ }^{43}$

Para la edición del tetrástico partiremos de los cuatro primeros testimonios conservados, que serían suficientes para establecer su texto; estos, además de $\mathbf{B}$ y $\mathbf{V}$, son dos ediciones granadinas de la versión abreviada del arte de Nebrija (Ga y Gb). A ellos añadiremos otros siete testimonios, a fin de determinar la posición del traslado boloñés (C) dentro de la tradición del tetrástico; así, nos valdremos de cuatro ediciones granadinas (Gc, Gd, Ge, Gf) y dos antequeranas (Aa, Ab) del arte abreviada de Nebrija; además, citaremos la segunda edición de los Elogia de Paolo Giovio (Elog), que también transmiten el poema que estamos estudiando.

$\mathbf{A a}=$ Antonio de Nebrija, Grammatica ([Antequera: Elio Antonio de Nebrija, 1573-1580] [edición no recogida en USTC]), f. [X6]r; véase Martín Baños 2014 (cit. n. 37), 228-229, no. 174.

$\mathbf{A b}=$ Antonio de Nebrija, Grammatica (Antiquariae: in aedibus D. Augustini Antonii Nebrissensis, 1597 [USTC 5045087]), f. [T2]r; véanse Odriozola 1946 (cit. n. 3), 23, no. 82; Esparza Torres, Niederehe 1999 (cit. n. 3), 176, no. 412; Martín Baños 2014 (cit. n. 37), 241-242, no. 199.

$\mathbf{B}=$ Antonio de Nebrija, Ars grammatica cum uberrimis commentariis ([Barcelona]: per Nicolaum Spindeler, 1505 [USTC 334592]).

Elog = Paolo Giovio, Elogia virorum literis illustrium (Basileae, Petri Pernae typographi Basil. opera ac studio, 1577 [USTC 683309]), 78. En ella, este poema responde al material añadido por Latomus en esta segunda edición; no se encuentra, en efecto, en la primera edición de la obra: Paolo Giovio, Elogia veris clarorum virorum imaginibus apposita (Venetiis, apud Michaelem Tramezinum, 1546 [USTC 833145]).

42 Antonio de Nebrija, Ad artem litterariam introductiones cum exactissima expositione (Venetiis: per Augustinum de Zannis de Portesio, 1512 [USTC 844358]); véanse Odriozola 1946 (cit. n. 3), 17, no. 27; Esparza Torres, Niederehe 1999 (cit. n. 3), 71-72, no. 98; Martín Baños 2014 (cit. n. 37), 127-128, no. 48. El tetrástico en honor de Nebrija se encuentra en el folio 160v, precedido, como en B, de los ocho dísticos anónimos.

43 Martín Baños 2014 (cit. n. 37), 330 (con lista de las ediciones que transmiten el tetrástico). 
Ga = Antonio de Nebrija, Grammatica (apud inclytam Garnatam, [Sancho y Sebastián de Nebrija], 1538 [USTC 844363]), f. [Tvii]v; véase Martín Baños 2014 (cit. n. 37), 192-193, no. 116.

$\mathbf{G b}=$ Antonio de Nebrija, Grammatica (apud inclytam Garnatam, per Xanthum Nebriss., 1540 [USTC 870337]), f. [Ti]v; véase Martín Baños 2014 (cit. n. 37), 194-195, no. 118.

Gc $=$ Antonio de Nebrija, Grammatica (apud inclytam Garnatam: per Xanthum Nebriss., 1550 [USTC 334655]), f. [Sviii]v; véanse Odriozola 1946 (cit. n. 3), 21, no. 61; Esparza Torres, Niederehe 1999 (cit. n. 3), 143, no. 317; Martín Baños 2014 (cit. n. 37), 209-210, no. 138.

Gd = Antonio de Nebrija, Grammatica (Granatensis [sic]: in aedibus Aeli Antonii Nebrissensis, 1581 [edición no recogida en USTC]), f. [X6]r; véase Martín Baños 2014 (cit. n. 37), 230, no. 179.

$\mathbf{G e}=$ Antonio de Nebrija, Grammatica (Granatae: in aedibus Aeli Antonii Nebrissensis, 1583 [edición no recogida en USTC]), f. [X4]r; véanse Odriozola 1946 (cit. n. 3), 23, no. 77; Esparza Torres, Niederehe 1999 (cit. n. 3), 166, no. 385; Martín Baños 2014 (cit. n. 37), 233, no. 184.

Gf = Antonio de Nebrija, Grammatica (Granatae: in aedibus Aeli Antonii Nebrissensis, 1590 [USTC 334593]), f. [X6]r; véanse Odriozola 1946 (cit. n. 3), 23, no. 80; Martín Baños 2014 (cit. n. 37), 237-238, no. 193.

$\mathbf{V}=$ Antonio de Nebrija, Ad artem litterariam introductiones cum exactissima expositione (Venetiis: per Augustinum de Zannis de Portesio, 1512 [USTC 844358]).

Ut quondam tacuit Latiae facundia linguae cum fuit Antoni caesa manus gladiis, sic scelus hoc solers Antoni cura piavit, quamque prior vitam sustulit, ille dedit.

Tit. Eidem tetrasthicon de eodem] B V : In eiusdem autoris laudem non indebitam. Tetrastichon Ga : In Antonii Nebrissensis laudem non indebitam. Tetrastichon (-con Ab) Gb Gc Aa Gd Ge Gf Ab : Incerti Elog : Io. Sepulu. in Aelium Nebris. grammat. C 1 Latiae] B V Gb Gc Aa Elog Gd Ge Gf Ab C : Latinae Ga 2 Antoni] B V Ga : Antonii Gb Gc Aa Elog Gd Ge Gf Ab C 3 Antoni] B V Ga Gb Gc : Antonii Aa Elog Gd Ge Gf Ab C 4 quamque] Ga Gb Gc Aa Elog Gd Ge Gf Ab C : quamquam B V | sustulit] B V Ga Gb Gc Aa Elog Gd Ge Ab : sustult Gf: sustutulit C | ille] Ga Gb Gc Aa Elog Gd Ge Gf Ab C : iste B V 
La traducción del tetrástico es la siguiente:44

Como en otro tiempo enmudeció la elocuencia de la lengua latina, cuando las espadas de Antonio le cortaron las manos, así el diligente cuidado de Antonio ha expiado este crimen, y la vida que el primero le arrebató, aquel ilustre varón se la devolvió. ${ }^{45}$

El epigrama es, ciertamente, ingenioso. Partiendo del relato de la muerte de Cicerón (transmitido en Sen. Rh. Suas. 6.17 [= Liv. frg. 59] y Plut. Cic. 47-49), el anónimo autor establece una contraposición entre (Marco) Antonio y Antonio (de Nebrija): el primero, mediante el asesinato de Cicerón, privó al latín de su elocuencia; el segundo, por medio de su obra gramatical, devolvió a la vida dicha lengua.

En cuanto a la técnica versificatoria, es de notar - como acertadamente uno de los revisores anónimos - la presencia de un trisílabo al final del primer pentámetro (gladiis), mientras que en los epigramas dedicados a Zúñiga y a Maximiliano de Austria aparecen siempre bisílabos en dicha posición. ${ }^{46}$ No es, tomado aisladamente, un indicio suficiente para rechazar la atribución a Sepúlveda del tetrástico, pero puede reforzar de algún modo la argumentación que desarrollaremos a continuación.

La abundancia de los testimonios disponibles nos permite reconstruir el proceso por el que se llegó a la forma corrupta de texto que transmite C, con las dos vistosas faltas contra metrum en los versos 2 y 3 ("Antonii

44 Otras traducciones de este epigrama se encuentran en V. Bonmatí Sánchez, "Las Laudes Nebrissensis en la edición Ad artem litterariam introductiones de Elio Antonio de Nebrija de Cristóbal Escobar, Venecia 1512”, Studia philologica Valentina 14 (2012), 171178, en concreto 177, n. 18; T. Jiménez Calvente, "Nebrija en los Virorum doctorum elogia de Paulo Jovio", Revista de Filología Española 74 (1994), 41-70, en concreto 53, n. 29; Martín Baños 2014 (cit. n. 37), 330.

45 No podemos dejar de mencionar dos cuestiones que, extrañamente, han causado ciertos problemas en algunas de las traducciones señaladas en la nota anterior: en el verso 2 manus es un acusativo de relación que depende del participio caesa; a su vez, en el verso 4 sustulit es el perfecto de tollo.

46 Sobre la preferencia de los poetas renacentistas por los finales bisílabos en los pentámetros, véase J. Pascual Barea, "Algunas particularidades de prosodia y métrica latinas del Renacimiento", en Luque Moreno, Díaz y Díaz 1999 (cit. n. 34), 747-766, concretamente 750-752. Para ilustrar el precepto, puede citarse, entre otros, a Giovanni Sulpizio da Veroli, De versuum scansione ([Roma, Stephan Plannck, 1483] [USTC 990719]), f. [35]r: "Pentametrum elegiacum sensum concludere debet suaviusque in vocem bissyllabam desinit; proxime in pentasyllabam tetrasyllabamque, pessime in trissyllabam et monosyllabam." No es, pues, que el autor del epigrama en honor de Nebrija se aparte del usus presente en los dos epigramas publicados por Sepúlveda, sino que comete una grave transgresión de la preceptiva humanística. 
[...] Antonii"). Para ello aceptaremos en primer lugar la propuesta de Martín Baños de que el tetrástico se hubiese publicado por primera vez en una edición castellana, hoy perdida, anterior a 1500: no puede explicarse de otra manera que Ga (el primer testimonio del tetrástico en ambiente castellano), aun siendo más de treinta años posterior a $\mathbf{B}$, conserve mejor la forma primitiva del texto. B, en efecto, testimonia dos faltas en el verso 4, ausentes de la tradición granadino-antequerana del tetrástico: la primera, muy llamativa: quamquam (escrito mediante compendio quamq) en lugar de quamque; la segunda, menos aparente: iste en lugar de ille. ${ }^{47}$ Por lo demás, que ambos errores aparezcan en $\mathbf{V}$ apoya la suposición de Martín Baños de que $\mathbf{V}$ toma este material de $\mathbf{B} .^{48}$

Con Ga comienza la tradición granadino-antequerana del tetrástico, independiente de $\mathbf{B} \mathbf{V}$, aunque derivada de la misma fuente (la edición castellana perdida del arte de Nebrija conjeturada por Martín Baños). Dentro de esta familia textual, la primera lección errónea Antonii (v. 2) se introduce ya en $\mathbf{G b}$, perpetuándose en las subsiguientes ediciones; a su vez, Aa suma al anterior error el Antonii del verso 3, dando lugar a la forma de texto transmitida, entre otros testimonios, por Elog y C. Por lo demás, teniendo en cuenta que la transmisión del tetrástico, dejando de lado $\mathbf{C}$, es exclusivamente impresa, hemos de considerar que este último dependa de Ab (o de una edición granadina o antequerana posterior) o de Elog, siendo más probable lo primero. En todo caso, la copia del tetrástico en C habría de datarse con posterioridad a 1573-1580 (fecha de Aa, que canoniza la forma de texto presente en $\mathbf{C}$ ).

Así pues, la atribución en $\mathbf{C}$ de este tetrástico a Sepúlveda constituye no solo un caso aislado, sino por ende muy tardío, dentro de la transmisión del poema. Por lo tanto, tal atribución ha de responder a una mera conjetura, nacida con gran probabilidad en el ambiente del Colegio de España: a este origen apunta, en efecto, que mediante aquella se unieran las figuras de dos ilustres colegiales.

No cabe, pues, atribuir a Sepúlveda la autoría del tetrástico en honor a Nebrija que figura en el folio $67 \mathrm{r}$ de $\mathbf{C}$ (transcrito, como hemos visto, por una mano que no es posible identificar con la del pozoalbense, pese a la

47 La lección "prior [...] ille" es, ciertamente, difficilior, puesto que en ella se combina un adjetivo con función fórica (prior, "el primero [que se ha mencionado]") con un pronombre con función enfática (ille, "aquel [ilustre varón]"). La lección "prior [...] iste" ("el primero [...] este"), exclusiva de $\mathbf{B}$ y $\mathbf{V}$, surge de la consideración de que tras prior, empleado como fórico, ha de aparecer otro fórico con valor proximidad, lo que justifica la aparición de iste en lugar de ille.

48 Véase Martín Baños 2014 (cit. n. 37), 327. 
afirmación de Lamadrid). Este, epigrama, así pues, debe considerarse la obra de un anónimo. ${ }^{49}$

\section{El epigrama en honor de Juan Domínguez de Paniza}

Pasando al epigrama In Ioannem Paniza, comenzaremos diciendo que su cronología puede determinarse fácilmente a partir de su contenido. En esta composición se agradece a Juan Domínguez de Paniza ${ }^{50}$ el regalo de un libro a la Biblioteca del Colegio de España en Bolonia: ${ }^{51}$ tuvo, pues, que ser escrita con tal ocasión, que debió de tener lugar durante la estancia del mencionado personaje en dicha institución (que se extendió desde el 7 de enero de $1514^{52}$ hasta poco después del 16 de marzo de 1524), ${ }^{53}$ primero como colegial y luego como capellán (esto último a partir del 29 de abril de 1522). ${ }^{54}$ Este cambio de situación de Paniza dentro del Colegio de España tiene su importancia para la fijación de la cronología de este poema: si damos crédito al tenor de la praescriptio en $\mathbf{B}$, ha de notarse que en ella se le da al destinatario del epigrama el apelativo de collega, es decir, "colegial"; de acuerdo con esto, el poema en honor de Paniza hubo de componerse entre su ingreso en el Colegio y su nombramiento como capellán (es decir, entre el 7 de enero de 1514 y el 29 de abril de 1522).

Aun cuando dudásemos del testimonio de $\mathbf{B}$ en este punto (puesto que M omite collegam), e incluso si rechazásemos la autenticidad de la praescriptio (atribuyendo su paternidad a Lamadrid), no se verían afectadas ni

49 Bonmatí Sánchez 2012 (cit. n. 44), 175 n. 13, a partir de la presencia del tetrástico en $\mathbf{V}$, se pregunta si su autor pudiera ser Cristóbal Escobar. Ahora bien, dado que este epigrama aparece por primera vez en $\mathbf{B}$, es de rechazar tal conjetura.

50 Sobre el clérigo zaragozano Juan Domínguez de Paniza, véase A. Pérez Martín, Proles Aegidiana, vol. 2 (Bolonia, 1979), 579-580, no. 653. Este personaje no parece haber dejado más rastro en la historia que su paso por el Real Colegio de España en Bolonia.

51 Concretamente, el ejemplar de la Gramática castellana de Nebrija, actualmente integrado en $\mathbf{C}$, en cuyo primer folio (hoy perdido), según el testimonio de Lamadrid, se encontraba manuscrito el presente epigrama.

52 El acta de admisión se encuentra en Archivo del Real Colegio de España, Libri admissionum, vol. 3, f. 46v.

53 La última referencia a Paniza en la documentación boloñesa se encuentra en Archivo del Real Colegio de España, Libri admissionum, vol. 3, f. 81v, en donde se deja constancia, con fecha de 16 de marzo de 1524, de la vuelta de Paniza al Colegio (había partido de él el 20 de febrero con la intención de visitar Roma y Loreto). A partir de este momento, su nombre desaparece de la documentación.

54 El acta de nombramiento se encuentra en Archivo del Real Colegio de España, Libri admissionum, vol. 3, f. 73v. 
la identificación del destinatario ni la datación del poema. Que el Paniza que dona un libro al Colegio de España en Bolonia sea Juan Domínguez de Paniza es una suposición bastante fundada: la única persona con dicho apellido relacionada con el Colegio es el personaje que estamos tratando. ${ }^{55}$ Por otra parte, ha de tenerse asimismo en cuenta que el nombramiento de Paniza como capellán en 1522 tenía un dudoso encaje estatutario, ya que podía entenderse como un fraude destinado a prolongar ilícitamente el período máximo de permanencia en el Colegio. Siendo ello así, difícilmente podría el autor del epigrama haber alabado en Paniza una actitud desinteresada, si este hubiera sido nombrado ya capellán, o si hubiese manifestado su deseo de solicitar tal puesto.

Para la edición del epigrama nos basaremos en las dos transcripciones de Lamadrid ( $\mathbf{M}$ y B), que son, como hemos mencionado repetidamente, los dos únicos testimonios conocidos de este poema. Regularizaremos su ortografía, omitiendo asimismo en el aparato las variantes meramente ortográficas.

Ioannis Gennesii Sepulveda in Ioannem Paniza collegam, qui hunc librum Bibliothecae addixit

Quod sis munificus, quod sis hac mente benignus muneribus nullum ut sollicitare velis,

hoc iuvat, hoc laudo, quippe est donatio vera: institor est praebens, maius ut accipiat.

Ergo non orbis, non das, Paniza, pupillis, munere sed gaudet Bibliotheca tuo.

Magna ferant dites, varie $<\mathrm{n}>\mathrm{t}$ emblemate nidos, archetypos addant fronte micante libros.

Te donare sat est parvum (pro tempore) munus exemploque alios admonuisse tuo.

Tit. Ioannis Gennesii] B : Ioannes Gennesius M | collegam] B : om. M. post tit., Epygramma B $\mathbf{2}$ nullum ut] B : ut nullum $\mathbf{M}$ | sollicitare] $\mathbf{M}$ : sollecitare B $\mathbf{7}$ uarie $<\mathrm{n}>\mathrm{t}]$ scripsi : uariet $\mathbf{B M}$

55 Esto puede comprobarse recorriendo los índices de P. Bertrán Roigé, Catálogo del Archivo del Colegio de España (Bolonia, 1981). 
Antes de entrar en cuestiones textuales, ofreceremos una traducción de este epigrama: ${ }^{56}$

De Juan Ginés de Sepúlveda al colegial Juan Paniza, que donó este libro a la Biblioteca. Que seas dadivoso, que seas espléndido con la intención de no querer ganarte a nadie mediante regalos, esto está bien, esto lo alabo, pues la donación es sincera: quien ofrece para recibir algo mayor es un mercader. Así pues, ${ }^{57}$ no das, Paniza, a unos desposeídos, no a unos pupilos; y, pese a ello, la Biblioteca se alegra de tu regalo. ${ }^{58}$ Ofrezcan grandes cosas los ricos, adornen con taraceas las librerías, ${ }^{59}$ añadan libros originales ${ }^{60}$ de brillante portada. ${ }^{61}$ Es suficiente que, por el momento, hagas un pequeño regalo y que hayas inspirado a otros con tu ejemplo.

En cuanto a la praescriptio del epigrama, hemos seguido el testimonio de B (rechazando, con todo, el subtítulo Epygramma), si bien como mera tentativa. En efecto: como hemos visto al comparar el texto de In Aelium Nebrissensem transmitido por $\mathbf{B}$ y $\mathbf{M}$ con el folio $67 \mathrm{r}$ de $\mathbf{C}(\$ 1)$, Lamadrid modifica a su gusto el título que precede a dicha composición en el antígrafo, añadiendo además en B, a modo de subtítulo, Epygramma teniendo esto último en cuenta, hemos supuesto que tal subtítulo responda también aquí a un añadido. A este respecto - y poniéndonos en el peor de los casos - podríamos incluso suponer que en $\mathbf{C}$ esta composición careciera de praescriptio y que Lamadrid se hubiese sacado de la manga el título que, con las variantes señaladas, aparece en $\mathbf{M}$ y B. Esta hipótesis, a

56 No nos consta la existencia de otra traducción de este poema.

57 Como puede observarse en la traducción, ergo no presenta aquí su originario valor ilativo. Cabría, pues, ver en el empleo de esta conjunción el "usus inceptivus et transitorius" que se describe en ThlL 5.2 773.15-774.67.

58 Los v. 5-6 resultan, como puede comprobarse, un tanto crípticos, debido al uso metafórico de los términos orbus y pupillus. El sentido de estos versos es el siguiente: Paniza dona un volumen a una institución cuyos integrantes no carecen de libros, como tampoco de criterio para juzgar su valor; y, pese a ello, el regalo de Paniza es sinceramente apreciado.

59 Sobre este significado de nidus, véase la quinta acepción registrada por C.T. Lewis, C. Short, Latin Dictionary (Oxford, 1956), s.v. nidus, "a receptacle, case, for books or goods"; cf. Mart. Epigr. 7.17.5 "Hos [sc. libros] nido licet inseras vel imo."

60 Para este significado técnico de liber archetypus, véase S. Rizzo, Il lessico filologico degli umanisti (Roma, 1973), 308.

61 Sobre frons con el significado de "portada" en el léxico humanístico, véase Rizzo 1973 (cit. n. 60), 41. 
nuestro entender, resulta demasiado artificiosa, e implicaría un profundo trabajo de investigación por parte de Lamadrid: así, aunque la deducción de que Paniza había sido un colegial pudiese haber resultado casi obvia, Lamadrid habría tenido que aventurar la fecha del poema para acudir a tiro cierto a los documentos de archivo pertinentes; estos le habrían permitido conocer el nombre propio del destinatario del poema (puesto que en este solo aparece su apellido), así como aventurar, a partir de la constatación de la convivencia en el Colegio de Paniza y Sepúlveda, que el autor del epigrama fuese este último. Ahora bien: aun admitiendo como factible todo lo anterior, resta el hecho de que las palabras "qui hunc librum Bibliothecae addixit" solo tienen sentido escritas en C, lo que lleva a pensar que Lamadrid encontró en él una praescriptio que contenía tales palabras. ${ }^{62} \mathrm{Si}$ en esta se mencionaba o no a Sepúlveda, esto es ya imposible de demostrar positivamente.

En lo referente al texto del epigrama, es evidente que en el verso 2 ha de seguirse, en principio, a B ("muneribus nullum ut"), pues el texto transmitido por $\mathbf{M}$ ("muneribus ut nullum"), y seguido por Losada, no encaja en el metro - como ya hemos apuntado en la n. 9 -; de este error de $\mathbf{M}$ fue ya consciente Casimiro Ortega, a quien la Real Academia de la Historia encargó en 1792 un informe sobre los documentos enviados por Lamadrid. ${ }^{63}$ El problema de la lección ofrecida por $\mathbf{B}$ es que coloca un monosílabo antes de la diéresis del pentámetro. Ahora bien, tal como hemos visto en $\$ 2$, remitiéndonos a las estadísticas de Serrano Cueto, tal monosílabo podría justificarse aduciendo que está unido mediante sinalefa a la palabra que le precede.

En cuanto al verso 7, hemos corregido el amétrico uariet (transmitido unánimemente por $\mathbf{B}$ y $\mathbf{M}$ ) en uarie $n>t$, haciéndolo así concordar con su sujeto, dites (nominativo plural de dis, ditis), que lo es también de ferant en el mismo verso y de addant en el siguiente.

62 Sería, en nuestra opinión, demasiado retorcido suponer que las citadas palabras respondiesen al deseo de Lamadrid de hacer pensar al lector que la mencionada praescriptio figuraba ya en C. En efecto: a partir de la documentación conservada en la Real Academia de la Historia y en el Colegio de España de Bolonia podría, quizás, sostenerse que Lamadrid era un erudito carente de método, pero en modo alguno que fuese un falsario.

63 El informe se conserva en la Biblioteca de la Real Academia de la Historia (ms. 96117-51); se encuentra editado en Losada 1949 (cit. n. 1), 601-602. Ortega, en efecto, hace notar que en el traslado de Lamadrid "la transposición de una palabra hace que no conste el segundo verso." 
En lo relativo a la prosodia, ha de señalarse un error: en la cláusula del v. 5, la sílaba inicial de pūpillis aparece escandida como breve. Esta transgresión no deja de ser llamativa, y ello por dos razones. En primer lugar, aunque la palabra pupillus está prácticamente ausente de la poesía clásica (véase ThlL 10.2 2661.43-44), se documenta, con todo, en pasajes que el autor del epigrama podía fácilmente conocer (por ejemplo, Hor. Ep. 1.1.22, 2.1.123; Pers. Sat. 2.12, 4.3; Iuv. Sat. 1.47, 6.629, 10.223, 15.135). En segundo, la cantidad de la primera sílaba de pupillus recibió la atención de la gramática humanística: así, por ejemplo, Nebrija cita dicha palabra entre las que constituyen una excepción a la regla prosódica " $u$ ante $p$ brevis est." 64 Por lo demás, merece únicamente señalarse la escansión del nombre Pānīză (tratado como un masculino de la primera declinación), y ello para subrayar que no es del todo arbitraria: la cantidad larga de la penúltima sílaba se justifica por el hecho de que $z$ hace posición (y también porque sobre tal sílaba recae el acento en castellano); a su vez, en lo tocante la cantidad larga de la primera sílaba, puede pensarse en una analogía (o, quizás incluso, una relación etimológica) con pānis. ${ }^{65}$

Pasando al contenido del epigrama, podemos decir que nos encontramos de nuevo ante una composición de circunstancias. Comienza su autor dando la vuelta a un tópico bien conocido desde la Antigüedad: quien hace grandes regalos pretende obtener a cambio grandes beneficios; este se encuentra, por ejemplo, en Mart. Epigr. 5.59.3 "quisquis magna dedit, voluit sibi magna remitti." Así, tras alabar la intención de Paniza en los versos 1-4 (no hace regalos para sacar rédito de ellos, actitud que sería propia de un mercader, institor), el autor da cuenta en los versos 5-6 del hecho digno de encomio llevado a cabo por el destinatario del epigrama: el regalo destinado a la Biblioteca del Colegio. Tal donación, aunque se califica como modesta, se juzga un ejemplo a seguir por otros (supuestamente, otros colegiales), más adinerados que Paniza (v. 7-10).

El parvum munus al que se hace referencia ha de identificarse, siguiendo la praescriptio del epigrama, con el ejemplar de la Gramática castellana en cuyo primer folio, hoy perdido, se encontraba transcrita esta composición. El entusiasmo que muestra el autor del epigrama por este "pequeño regalo" se justifica por su valor simbólico:en los primeros decenios del s. XVI, Antonio de Nebrija se convirtió en un señalado

64 Antonio de Nebrija, Introductiones Latinae (Salmanticae, [Juan de Porras], 1495 [USTC 766686]), f. [148v].

65 Sobre procedimientos similares para la escansión de nombres propios contemporáneos, véase Maestre Maestre 1999 (cit. n. 34), 575-576. 
referente para Colegio de España, como testimonia el propio Sepúlveda en la Brevis Collegii descriptio 5.4, en donde coloca al gramático a la cabeza de los excolegiales ilustres. Durante este período el Colegio trató de hacerse con las obras de su ilustre alumno, ${ }^{66}$ circunstancia esta que explica que la donación de Paniza fuese especialmente estimada y se la juzgase merecedora de un epigrama. Por lo demás, aunque el regalo de Paniza se califique como modesto - y quizás lo fuese desde un punto de vista meramente pecuniario -, la Gramática castellana no debía de ser fácil de conseguir: como es bien conocido, la publicación de esta obra pasó sin pena ni gloria, como prueba el hecho de haber tenido una única edición, que sin duda fue de tirada modesta, habida cuenta de que solo se conservan escasísimos ejemplares de la misma.

La pregunta que cabe hacerse ahora es si el epigrama en honor de Paniza puede atribuirse a Juan Ginés de Sepúlveda. Dado que, a fin de solventar esta cuestión, no podemos apelar ni a la declaración de autoría presente en la praescriptio de este poema, ni a su supuesta autografía en C, como tampoco llevar a cabo una comparación con el resto de los poemas del pozoalbense (dado que su reducida extensión no permite hacer generalizaciones estilísticas), solo nos queda acudir a criterios externos.

Partiremos del hecho de que Paniza y Sepúlveda convivieron en el Colegio de España. ${ }^{67}$ A la estancia en él del primero ya nos hemos referido en precedencia; de Sepúlveda sabemos que ingresó en el Colegio el 27 de junio de $1515,{ }^{68}$ permaneciendo en él, con las ausencias que a continuación señalaremos, hasta el 6 de mayo de 1523. Ahora bien: un dato relevante es que la convivencia de Sepúlveda con Paniza tuvo lugar casi exclusivamente antes del nombramiento de este último como capellán. El pozoalbense, desde el momento de su admisión en el Colegio, residió permanentemente en él hasta el 28 de febrero de 1522, cuando parte para

66 Véase A. García y García, A. Domingo Malvadí (ed., tr.), Antonio de Nebrija, Annotationes in libros Pandectarum (Salamanca, 1996), 17.

67 Sobre este período de la vida de Sepúlveda, véanse Losada 1949 (cit. n. 1), 37-48; Pérez Martín 1979 (cit. n. 50), 604-606, no. 670; S. Muñoz Machado, Biografía de Juan Ginés de Sepúlveda (Pozoblanco, 2012), 47-59.

68 El acta se conserva en Archivo del Real Colegio de España, Libri admissionum, vol. 3, f. $52 \mathrm{v}$. 
Carpi - sin duda, para visitar al príncipe Alberto Pío. ${ }^{69}$ Retorna a Bolonia el 17 de marzo, ${ }^{70}$ pero el 8 de abril vuelve a ausentarse (en principio, por un mes y medio). ${ }^{71}$ Entretanto, tiene lugar la elección como capellán de Paniza (29 de abril), en la que Sepúlveda no está presente. Su ausencia se prolonga hasta el 4 de abril de $1523 ;^{72}$ y, poco más de un mes después, el 6 de mayo, ${ }^{73}$ pide de nuevo licencia para ausentarse del Colegio por dos meses, que transcurrieron sin que Sepúlveda volviese a él. En consecuencia, en octubre de 1523 se declaró vacante su plaza. ${ }^{74}$ Así pues, de haber sido compuesto el epigrama a Paniza mientras este ocupó en Bolonia una plaza de colegial (como hemos sostenido basándonos en su praescriptio y, sobre todo, en las implicaciones de su contenido), nada más natural que el Colegio encargase a Sepúlveda, que por entonces residía de continuo en él, la composición de dicho poema. En este sentido cabe recordar que, habida cuenta de las dotes de Sepúlveda como latinista, el Colegio le

69 Archivo del Real Colegio de España, Libri admissionum, vol. 3, f. 73r "Die ultima Februarii D. Ioannes Sepulveda, rectore et consiliariis permittentibus, Collegio recedit iturus in oppidum del Carpio [sic] pro negotiis suis. Iacobus Pontius rector scripsi." Durante este período de permanencia continua de Sepúlveda en el Colegio (27 de junio de 1515 - 28 de febrero de 1522), Paniza solo se ausentó brevemente, del 5 al 14 de mayo de 1521, para viajar a Venecia (Archivo del Real Colegio de España, Libri admissionum, vol. 3, f. 71r).

70 Archivo del Real Colegio de España, Libri admissionum, vol. 3, f. 73r "Die 17 Martii D. Io. Sepulveda revertitur."

71 Archivo del Real Colegio de España, Libri admissionum, vol. 3, f. 73v "Die 8 Aprilis D. Ioannes Sepulveda, habita licentia a rectore et D. Iohanne Paniza et D. Iacobo Castillo de Villasante consiliariis, Collegio recessit, praestito prius iuramento, secundum statutorum formam, quod urgente iusta causa recedebat et erat iuxta possibilitatem ad electionem reversurus; reliquit tamen procuratorem suum D. Iacobum Castillo de Villasante. Iacobus Pontius rector scripsi." Las elecciones que se mencionan son las que se celebraban cada año el 1 de mayo para el nombramiento del rector del Colegio. Nótese la presencia de Paniza como consiliario.

72 Archivo del Real Colegio de España, Libri admissionum, vol. 3, f. 78r "Die 4 aprilis revertitur in Collegium D. Io. Sepulveda. Ita est. Antonius Zarate vice rector."

73 Archivo del Real Colegio de España, Libri admissionum, vol. 3, f. 79r "Die 6 maii D. Io. Sepulveda Collegio discedit reversurus infra duos menses, petita prius et obtenta a me, Antonio Zarate, Collegii rectore, licentia. Constituit in omnibus suum procuratorem et fidei iussorem D. Franciscum Mota. Ita est. Antonius Zarate rector."

${ }^{74}$ Archivo del Real Colegio de España, Libri admissionum, vol. 3, f. 80r "Quia Dns. Ioannes Sepulveda fuit absens a Collegio ultra annum cum dimidio, licet in diversis vicibus, ideo titulus eius vacat $\mathrm{S}$. Ecclesiae Toletanae, ut statuto cavetur, quod in toto octonario nullus possit esse absens a Collegio ultra annum cum dimidio. Antonius Zarate Collegii rector." Aunque está entrada no tiene fecha, ha de datarse entre el 11 y el 28 de octubre de 1523, habida cuenta de las anotaciones que la preceden y la siguen. 
encargó por este mismo tiempo la redacción del Liber gestorum Aegidii Albornoti (publicado en 1521). ${ }^{75}$

\section{Conclusiones sobre la atribución a Sepúlveda de los epigramas In Aelium Nebrissensem e In Paniza}

De los dos epigramas que Losada publicó, atribuyéndolos a Juan Ginés de Sepúlveda, el dedicado a Nebrija es sin duda alguna obra de un anónimo. Su copia y atribución al pozoalbense debió de tener lugar en el ambiente del Colegio de España, ciertamente con posterioridad a 1573-1580.

En lo que toca al epigrama en honor de Paniza, nada impide, en nuestra opinión, que pueda ser de Sepúlveda. Como hemos visto, en etapas posteriores de su vida compuso versos de ocasión (los epigramas dedicados a Zúñiga y a Maximiliano de Austria), mostrándose capaz de escribir versos, cuando menos, aceptables formalmente. Ahora bien, la corta extensión de los dos poemas incluidos en el Epistolario, su disparidad temática y su lejanía en el tiempo hace imposible llevar a cabo una comparación que pueda dirimir la cuestión de la autoría de In Paniza. Así pues, habrá que considerar cautamente que esta última composición es de atribución probable, habida cuenta los avatares de su transmisión, de la coincidencia de Paniza y Sepúlveda en el Colegio de España y de las circunstancias externas que enmarcaron la composición del epigrama.

Universidad de Almeria ramong@ual.es

75 De este encargo da cuenta el propio Sepúlveda, Liber gestorum Aegidii Albornotii, praef. 3.1-4. 


\section{Figuras}

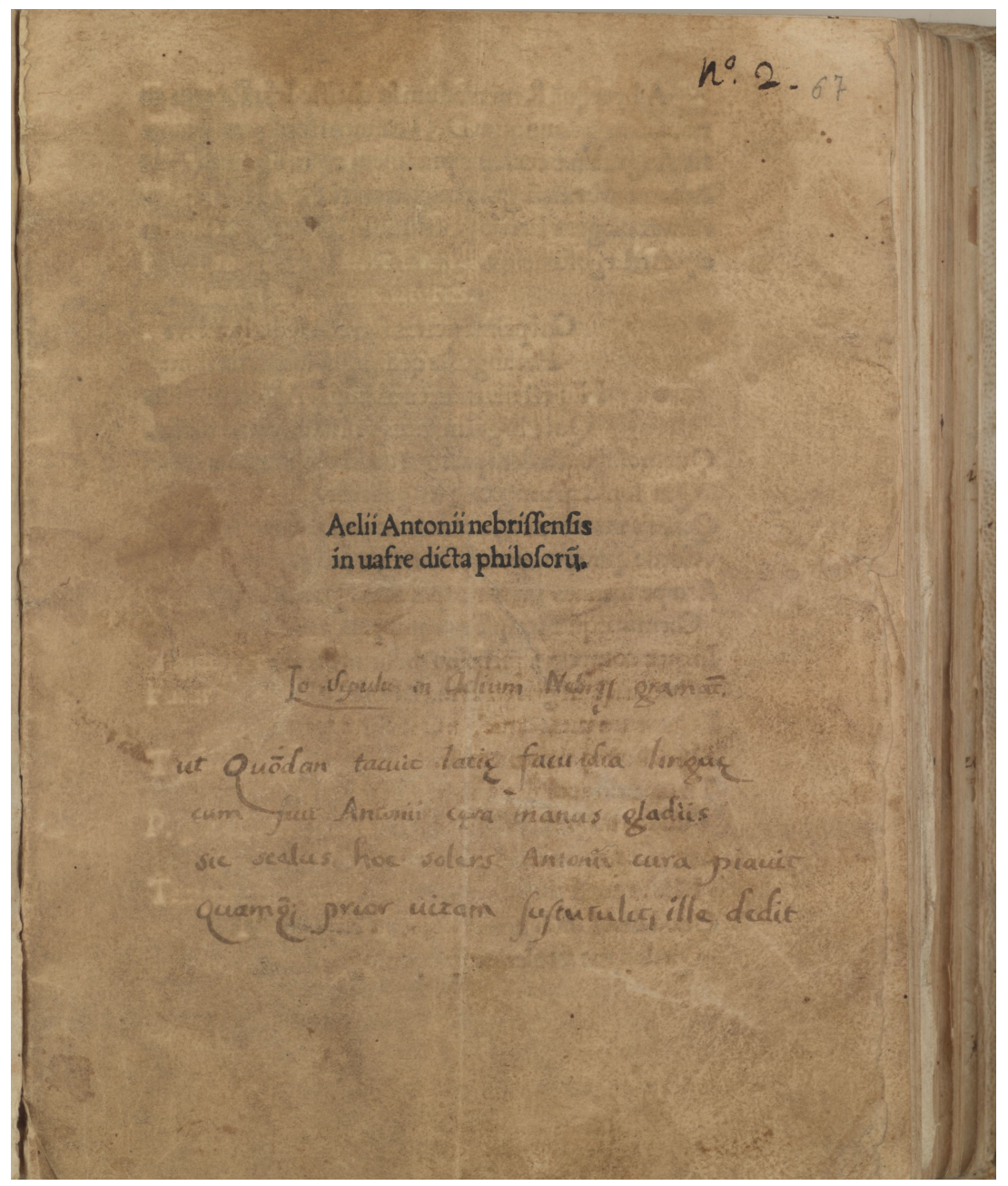

Fig. 1: Bologna, Biblioteca del Real Colegio de España, Cod. 132, f. 67r. 